\title{
Work posture analysis of activities performed by blacksmiths engaged in hand tools manufacturing units
}

Mira Kalita, Nandita Bhattacharyya and Ruplekha Borah

Received: 14.11.2017; Revised: 08.05.2018; Accepted: 25.05.2018

See end of the paper for authors' affiliations Mira Kalita

Department of Family Resource Management, College of Home Science, Assam Agricultural University, Jorhat (Assam) India Email : mirakalita72@yahoo. com
ABSTRACT : Repetitive processes and manual material handling are the major problems in hand tool manufacturing units. The presents study is focused on posture analysis of the workers working in hand tools manufacturing units of Jorhat district of Assam. For the purpose RULA (Rapid Upper Limb Assessment) was used to assess specific body postures of the workers and recommend the changes to be made in the body postures while working. The impact of posture was studied by using eye hand co-ordination and motor speed of the workers, which was measured by using Grooved Pegboard test. The prevalence of WMSDs was studied by using NMQ. The study was conducted on 30 blacksmiths engaged in various processes during manufacturing of hand tools. The photographs of different sections like preparing sheet, molding, hammering, sharpening of tools etc. showing different movements of the workers during performance of activities was observed and both side of the body was assessed. The results of RULA showed that majority of the (75\%) blacksmiths were working in medium risk level which requires further investigation and changes to be made soon in work postures for improving their health and wellbeing. About 25 per cent of the workers were found in high risk level that requires changes to be brought immediately for the benefit of the workers after proper investigation. The postural assessment of the workers at different stages observed that the neck, trunk at flexion; upper arm at flexion and extension; lower arm at pronation and wrist in neutral position. Changes were observed in Grooved Pegboard test before and after the activities. Proper rest periods were recommended to avoid WMSDs of the workers in the future.

KEY WORDS: Posture analysis, RULA, Risk levels, WMSDs, NMQ

- HOW TO CITE THIS PAPER : Kalita, Mira, Bhattacharyya, Nandita and Borah, Ruplekha (2018). Work posture analysis of activities performed by blacksmiths engaged in hand tools manufacturing units. Asian J. Home Sci., 13 (1) : 423-428, DOI: 10.15740/HAS/AJHS/13.1/423-428. Copyright@ 2018: Hind Agri-Horticultural Society. 